BMC

Genomics

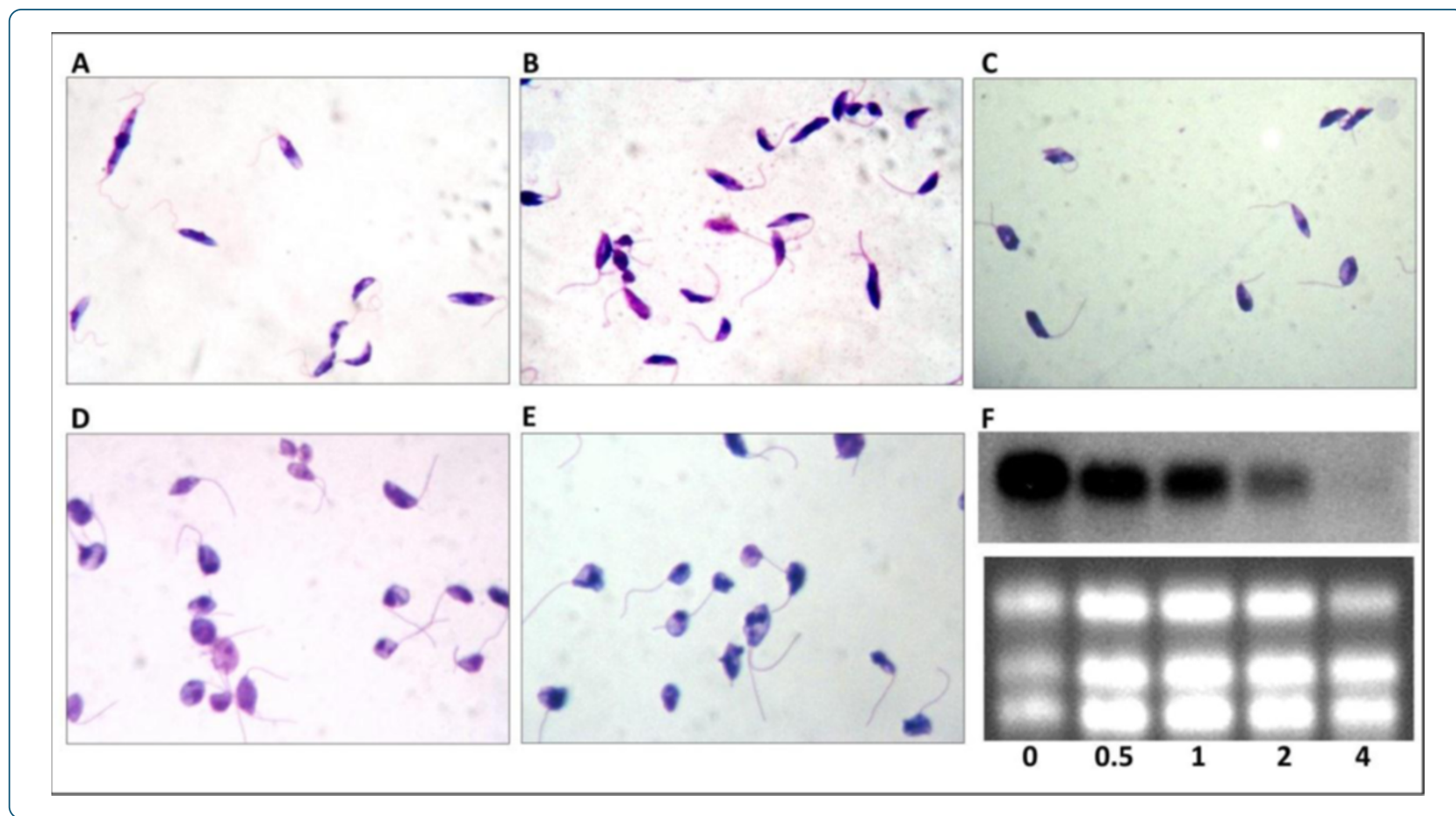

Alpha tubulin genes from Leishmania braziliensis: genomic organization, gene structure and insights on their expression

Ramírez et al. 


\title{
Alpha tubulin genes from Leishmania braziliensis: genomic organization, gene structure and insights on their expression
}

\author{
César A Ramírez ${ }^{1,2}$, José M Requena² and Concepción J Puerta ${ }^{1 *}$
}

\begin{abstract}
Background: Alpha tubulin is a fundamental component of the cytoskeleton which is responsible for cell shape and is involved in cell division, ciliary and flagellar motility and intracellular transport. Alpha tubulin gene expression varies according to the morphological changes suffered by Leishmania in its life cycle. However, the objective of studying the mechanisms responsible for the differential expression has resulted to be a difficult task due to the complex genome organization of tubulin genes and to the non-conventional mechanisms of gene regulation operating in Leishmania.
\end{abstract}

Results: We started this work by analyzing the genomic organization of $\alpha$-tubulin genes in the Leishmania braziliensis genome database. The genomic organization of $L$. braziliensis $\alpha$-tubulin genes differs from that existing in the L. major and L. infantum genomes. Two loci containing $\alpha$-tubulin genes were found in the chromosomes 13 and 29, even though the existence of sequence gaps does not allow knowing the exact number of genes at each locus. Southern blot assays showed that $\alpha$-tubulin locus at chromosome 13 contains at least 8 gene copies, which are tandemly organized with a 2.08-kb repetition unit; the locus at chromosome 29 seems to contain a sole $\alpha$-tubulin gene. In addition, it was found that L. braziliensis $\alpha$-tubulin locus at chromosome 13 contains two types of $\alpha$-tubulin genes differing in their $3^{\prime}$ UTR, each one presumably containing different regulatory motifs. It was also determined that the mRNA expression levels of these genes are controlled by post-transcriptional mechanisms tightly linked to the growth temperature. Moreover, the decrease in the $\alpha$-tubulin mRNA abundance observed when promastigotes were cultured at $35^{\circ} \mathrm{C}$ was accompanied by parasite morphology alterations, similar to that occurring during the promastigote to amastigote differentiation.

Conclusions: Information found in the genome databases indicates that $\alpha$-tubulin genes have been reorganized in a drastic manner along Leishmania speciation. In the L. braziliensis genome database, two loci containing $\alpha$-tubulin sequences were found, but only the locus at chromosome 13 contains the prototypic $\alpha$-tubulin genes, which are repeated in a head-to-tail manner. Also, we determined that the levels of $\alpha$-tubulin mRNAs are down-regulated drastically in response to heat shock by a post-transcriptional mechanism which is dependent upon active protein synthesis.

Keywords: Leishmania braziliensis, $\alpha$-tubulin, Expression, Untranslated region

\footnotetext{
* Correspondence: cpuerta@javeriana.edu.co

'Laboratorio de Parasitología Molecular, Departamento de Microbiología, Facultad de Ciencias, Pontificia Universidad Javeriana, Carrera 7 No. 43-82, Edificio 52, Oficina 608, Bogotá, Colombia

Full list of author information is available at the end of the article
} 


\section{Background}

Alpha tubulin, a highly conserved protein along the eukaryotic evolutionary tree, interacts with $\beta$-tubulin conforming an $\alpha / \beta$-tubulin heterodimer that comprises the structural subunit of microtubules, the basic building structure of the cytoskeleton, which is responsible for cell shape and is involved in many essential processes, including cell division, ciliary and flagellar motility and intracellular transport [1]. In unicellular microorganisms the cell shape and form are particularly relevant and depend on morphogenetic processes affecting essentially to the internal cytoskeleton. On the other hand, these microorganisms, and particularly the trypanosomatid flagellates, are very adequate models for studying the morphogenetic processes mediated by cytoskeleton reorganizations [2], as many aspects of their biology (infectivity and transmission) depend on these processes.

The Leishmania genus comprises at least 20 Leishmania species that infect humans, and the spectrum of diseases that they cause can be categorized broadly into three types: self-healing cutaneous leishmaniasis (CL), mucocutaneous leishmaniasis (MCL), and the often fatal visceral leishmaniasis (VL) [3]. Endemic leishmaniasis transmissions have been reported in 98 countries on 5 continents, and around two million new cases of leishmaniasis occur each year [4]. There are two major developmental forms in Leishmania, the motile promastigote and the amotile amastigote. Classical studies demonstrated that the promastigote stage synthesizes more tubulin protein than the amastigote stage, and that this biosynthetic change of tubulin was found to correlate with the morphological change of microtubules in leishmanial flagella and cytoskeleton during promastigote-to-amastigote transformation [5]. Studies aimed to uncover the regulation responsible for the differential expression of tubulin genes were initiated shortly after [6], but this has resulted to be a difficult task due to the complex genome organization of tubulin genes [7], in particular, and to the non-conventional mechanisms of gene regulation operating in Leishmania [8].

In various Leishmania species, the genomic organization of $\alpha$ - and $\beta$-tubulin genes has been analyzed, showing the existence of multiple copies, both arranged in tandem (forming separate clusters of $\alpha$ - and $\beta$-tubulin genes) and dispersed in the genome $[7,9,10]$. The availability of the genome sequences for several Leishmania species [11-13] has allowed resolving questions regarding the genome organization of complex gene families. In a recent work, Jackson and co-workers have carried out a comprehensive study about genomic organization of $\beta$-tubulin genes in several Leishmania species; these authors suggest that the gene organization has evolved to satisfy a need for innovative expression for $\beta$-tubulin genes [9].
In this work, we studied the organization of $\alpha$-tubulin genes in Leishmania braziliensis based on the available, yet incomplete, genome sequence. A comparison with the organization of this gene in L. infantum and L. major is also provided. The 5' and 3' untranslated regions (UTRs) for the different $\alpha$-tubulin genes in L. braziliensis have been determined as well as their mRNA expression levels under different conditions.

\section{Results and discussion}

Genomic organization of $\alpha$-tubulin genes in L. braziliensis, L. infantum and L. major

In the genome of Leishmania major (MHOM/IL/81/ Friedlin), the first Leishmania genome that was sequenced [13], a sole $\alpha$-tubulin locus exists. According to the data available at the GeneDB database [14], the locus is located at chromosome 13 and contains twelve copies (LmjF.13.0280 to LmjF.13.0390) having identical ORFs both in sequence and length (1356 bp), arranged in a head-to-tail tandem organization (Figure 1A). A similar arrangement, containing two copies (LmxM.13.0280 and LmxM.13.0390) separated by a sequence gap, and located also at chromosome 13, was found in the GeneDB database for Leishmania mexicana (MHOM/GT/2001/ U1103) genome. In contrast, according to the genome database (GeneDB), the Leishmania infantum (MCAN/ ES/98/LLM-877) genome contains two $\alpha$-tubulin loci, both located at chromosome 13 and separated by a region of $436.6 \mathrm{~kb}$. The more $5^{\prime}$ locus (Figure 1A) contains only a gene copy (LinJ.13.0330, ORF: 1356 bp), whereas the other locus (Figure 1B) has a complete copy (LinJ.13.1460, ORF: 1356 bp) and a truncated one (LinJ.13.1450, ORF: 708 bp). The genome of Leishmania (Viannia) braziliensis (MHOM/BR/75/M2904) [13], causative agent of CL and MCL in the New World [15], also contains two loci, one located at chromosome 13 and the other at chromosome 29. The locus at chromosome 13 (Figure 1A) is composed by two complete copies (LbrM.13.0190 and LbrM.13.0200, ORFs: 1356 bp) and an $\alpha$-tubulin-like gene (LbrM.13.0210, ORF: $702 \mathrm{bp}$ ). The locus at chromosome 29 (Figure 1A) contains a sole copy consisting of a truncated form of the $\alpha$-tubulin gene (LbrM.29.2700, ORF: 780 bp).

Comparison of the genomic region around the $\alpha$-tubulin loci in L. major, L. infantum and L. braziliensis revealed that the chromosomal regions surrounding the $\alpha$-tubulin genes at chromosome 13 have experienced remarkable reorganizations in these three Leishmania species, and the $\alpha$-tubulin locus itself appears as the target of DNA deletion and inversion events (Figure 1A). Thus, between L. major and L. infantum an inversion including the LmjF.13.0270 gene (colored in yellow in Figure 1A), the $\alpha$-tubulin locus (LmjF.13.0280 toLmjF.13.0390, colored in red in Figure 1A) and the LmjF.13.400 to LmjF.13.450 genes (colored in 


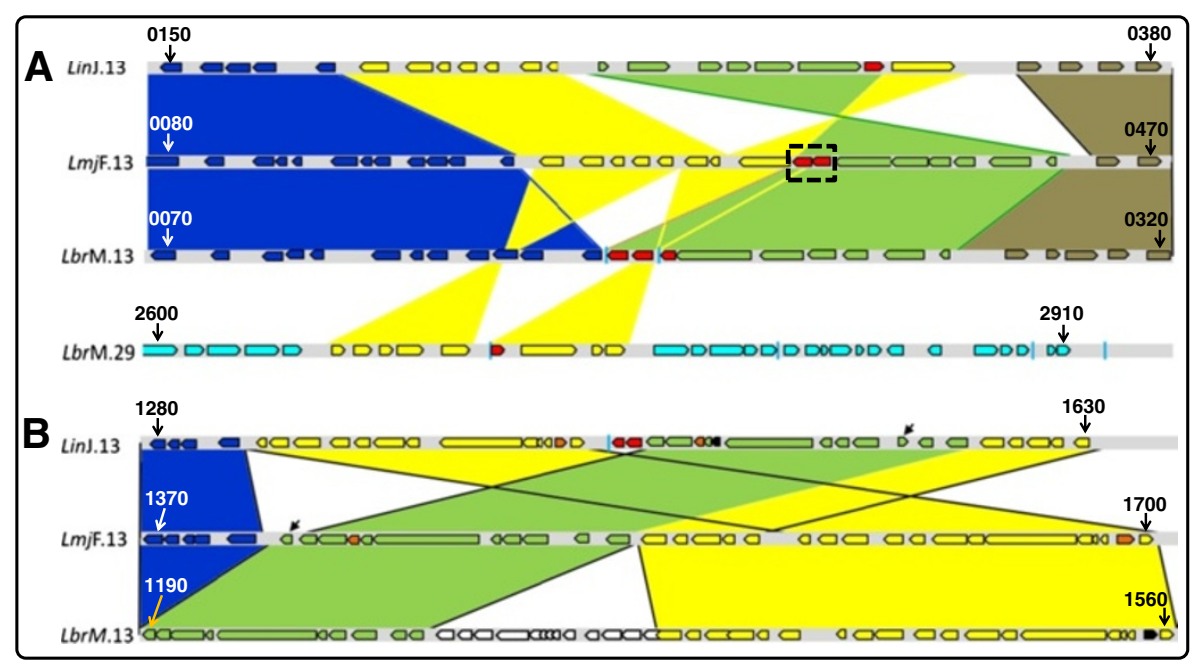

Figure 1 Genomic organization of $\boldsymbol{\alpha}$-tubulin genes and surrounding regions in three Leishmania species. Panel A: comparison among L. braziliensis (LbrM) $\alpha$-tubulin loci and syntenic regions in L. infantum (LinJ) and L. major ( LmjF) genomes. Panel B: comparison among the specific L. infantum $\alpha$-tubulin locus and the syntenic regions of L. braziliensis and L. major, species in which this locus is absent. Red boxes represent the $\alpha$-tubulin genes. L. major $\alpha$-tubulin locus contains 12 genes (dashed rectangle). Each block of syntenic genes in the different Leishmania species, and reorganization events affecting them, is colored differently. The diagonal arrow, point to the LinJ.13.1560 that has an inverted orientation regarding the homologous gene in L. major (B). Blue vertical bars denote gaps in sequence. Numbers in bold at the start and end of each gene cluster are the last four digits of GeneDB gene entries.

green in Figure 1A) took place. As a result of this event, the ORF polarity of the LinJ.13.0270 to LinJ.13.0320, $\alpha$-tubulin (LinJ.13.0330) and LinJ.13.0340 genes was changed with respect to the equivalent genes of L. major. In $L$. braziliensis, regarding the L. major chromosomal organization, a region spanning from the LmjF.0200 to the LmjF.0270 gene, corresponding to a string of 8 genes encoding for 7 hypothetical proteins and one putative class 3 lipase (colored in yellow in Figure 1A), was deleted. These deleted genes are present at chromosome 29 (LbrM.29.2650 to LbrM.29.2730, colored in yellow in Figure $1 \mathrm{~A}$ ) in the L. braziliensis genome; this reorganization that seems the result of two transposition events dragged a region of the $\alpha$-tubulin locus, generating the LbrM.29.2700 gene (colored in red in Figure 1A), which contains a truncated sequence of the prototypical $\alpha$ tubulin gene (see below).

The other $\alpha$-tubulin locus at L. infantum chromosome 13. comprising as mentioned above a partial and a complete copy of $\alpha$-tubulin coding sequence (Figure 1B), is found in a region that also has experienced clear reorganizations in these three Leishmania species. Thus, the regions flanking the $\alpha$-tubulin genes LinJ.13.1450 and LinJ.13.1460 in L. infantum chromosome have experienced a substantial reorganization regarding the equivalent chromosomal region in L. major, and again the $\alpha$ tubulin locus (absent in L. major) appeared as the center of the reorganization events (Figure 1B). Another minor transposition event, regarding the LinJ.13.1560 gene (signaled by a diagonal arrow in Figure 1B) took place after separation of these two Leishmania species. This chromosomal region in L. braziliensis (Figure 1B) is very similar to the $L$. major homologous region $(\alpha$-tubulin genes are also absent), excepting an insertion comprising the LbrM.13.1250 to LbrM.13.1370 gene block (Figure 1B). The LbrM.13.1250-LbrM.13.1370 orthologous are located on chromosome 34 in both $L$. major and L. infantum (data not shown).

In summary, these analyses pointed out that the $\alpha$ tubulin gene loci have been reorganized in a drastic manner along Leishmania speciation. However, this fact contrasts with the chromosomal organization of the three $\beta$-tubulin loci that is largely syntenic in $L$. major, L. infantum and L. braziliensis [9].

Nevertheless, it cannot be excluded the possibility that differences in the chromosomal distribution and organization of $\alpha$-tubulin genes among L. major, $L$. infantum and L. braziliensis are the result of artifacts generated during assembling of contigs into chromosomal scaffolds. The L. infantum and L. braziliensis genome sequences were produced by whole-genome shotgun sequencing to five- and six-fold coverage, respectively [12]. The resulting assemblies of L. infantum and L. braziliensis yielded 470 and 1,031 contigs, respectively. Finally, chromosomal scaffolds were produced by aligning contigs against the reference $L$. major sequence [13]. However, sequence gaps remain to be determined in the genome sequences for both $L$. infantum and $L$. braziliensis (according to the GeneDB database). Remarkably, the $\alpha$-tubulin locus at chromosome 29 in $L$. 
braziliensis (Figure 1A) and one of the $\alpha$-tubulin loci at chromosome 13 of L. infantum (Figure 1B) are located at the end of contigs (next to sequence gaps).

\section{Copy number determination in the L. braziliensis $\alpha$-tubulin loci}

As shown in Figure 1, the genomic regions containing the two $\alpha$-tubulin loci in chromosomes 13 and 29 of L. braziliensis has not been sequenced in full; there are sequence gaps that do not permit deducing the number of genes present at each locus. Moreover, tandem gene arrays are among the most challenging to resolve correctly using current genome sequencing technologies, since repetitive sequence reads tend to collapse into a single contig when no variation exists to distinguish them. Previous studies in L. major [16] and L. enriettii [10] showed the existence of tandem arrangements of $\alpha$-tubulin genes; in fact, the L. major genome database includes twelve gene copies tandemly arranged at chromosome 13. In order to determine the number of genes and their organization in the two $\alpha$-tubulin loci of L. braziliensis, Southern blots containing DNA digested with selected restriction enzymes were hybridized with a probe derived from the $\alpha$-tubulin coding region (Figure 2). Southern blot analysis of genomic DNA, partially digested with the Csp451 restriction enzyme, evidenced the typical ladder of tandemly repeated genes (Figure 2A). At least 8 copies of the repeated unit ( $2.08 \mathrm{~kb}$ in length) were observed. According to the restriction maps of the two $\alpha$-tubulin loci (Figure 2B, 2C and 2D), this tandem should be present in the locus at chromosome 13, whereas the locus at chromosome 29 would contain a sole $\alpha$-tubulin gene (see the hybridization band pointed by the triangle in Figure 2A, and $2 \mathrm{~B}$ ). Recently, Rogers et al. [11], analyzing Illumina highthroughput sequencing data, inferred that L. braziliensis genome should contain $15 \alpha$-tubulin copies (haploid content). Tandemly repetition of genes has been proposed as a direct mechanism for increasing the transcript abundance of highly expressed proteins [8], and it is known that $\alpha$-tubulin is an abundant protein in Leishmania [2].

When L. braziliensis total DNA was digested with restriction enzymes lacking cut sites within the $\alpha$-tubulin loci (PstI and ApoI), and the hybridization pattern was analyzed by Southern blotting, a conspicuous result was obtained (Figure 2B). The presence of three (Pst $\mathrm{I})$ and two (ApoI) large bands was unexpected. An explanation to this finding may be either the existence of allelic sequence polymorphisms in the regions surrounding the $\alpha$-tubulin locus at chromosome 13 or different copy numbers of $\alpha$-tubulin genes in each homologous chromosome. Indeed, similar observations have been described for L. braziliensis and other trypanosomatids regarding $H S P 70, K M P-11$ and histone $H 2 A$ gene loci [17-19]. Moreover, the presence of three hybridization bands in the lane containing PstI digested-DNA would be indicating that L. braziliensis has three homologous for chromosome 13, a very plausible possibility taking into account recent studies showing that L. braziliensis genome is essentially triploid [11].

\section{Sequence analysis of the $\alpha$-tubulins in L. braziliensis}

The genomic analysis described above indicated that the L. braziliensis $\alpha$-tubulin genes are encoding for three different amino acids polypeptides: the prototypical $\alpha$-tubulin (encoded by genes LbrM.13.0190 and LbrM.13.0200) and two $\alpha$-tubulin variants, dubbed $\alpha$-tubulin-A (LbrM.13.0210) and $\alpha$-tubulin-B (LbrM.29.2700) (Figure 3). The prototypical $\alpha$-tubulin is a 451 amino acid protein with a molecular weight of $49.7 \mathrm{kDa}$ and an isoelectric point of 4.9 . In contrast, the $\alpha$-tubulin-A is a shorter sequence with 233 amino acids, molecular weight of $24.9 \mathrm{kDa}$ and isoelectric point of 6.9. Similarly, the $\alpha$-tubulin-B sequence contains 259 amino acids, has a molecular weight of $28.8 \mathrm{kDa}$ and an isoelectric point of 5.0. As shown in Figure 3 , the $\alpha$-tubulin-A is identical to the $\alpha$-tubulin in the $81 \mathrm{~N}$-terminal amino acids, but they are absolutely divergent in the rest of the sequence. An intriguing finding was the observation of a remarkable sequence identity (100\%) between the divergent region of gene LbrM.13.0210 and a genomic region of chromosome 28 , located at the intercoding region of genes LbrM.28.2580 and LbrM.28.2590 (see Additional file 1).

In contrast, $\alpha$-tubulin-B sequence, excluding the $58 \mathrm{~N}$ terminal amino acids, was found to be identical to the C-terminal half of the prototypical $\alpha$-tubulin. Also, this $\alpha$-tubulin protein variant may have an extended sequence at the N-terminal region, since the LbrM.29.2700 annotated gene is close to a gap in the genomic sequence of the L. braziliensis genome database (Figure 1A).

As mentioned above, if assembly artifacts exist in the L. braziliensis genome database, the real existence of the $\alpha$-tubulin variants encoded by genes LbrM.13.0210 and LbrM.29.2700 may be questioned. To elucidate this, we have searched in the literature for experimental evidence of these genes or their products. The sole reference found is their presence in the gene lists generated by Rogers and co-workers [11] when analyzed the copy number of genes along the genome of L. braziliensis. However, this is not a demonstration of the real existence of these genes as the copy number was determined by mapping Illumina reads to the reference genome [12], but it was not analyzed whether the reads map homogenously along the gene ORF. On the other hand, none of these genes have homologs in the L. braziliensis 


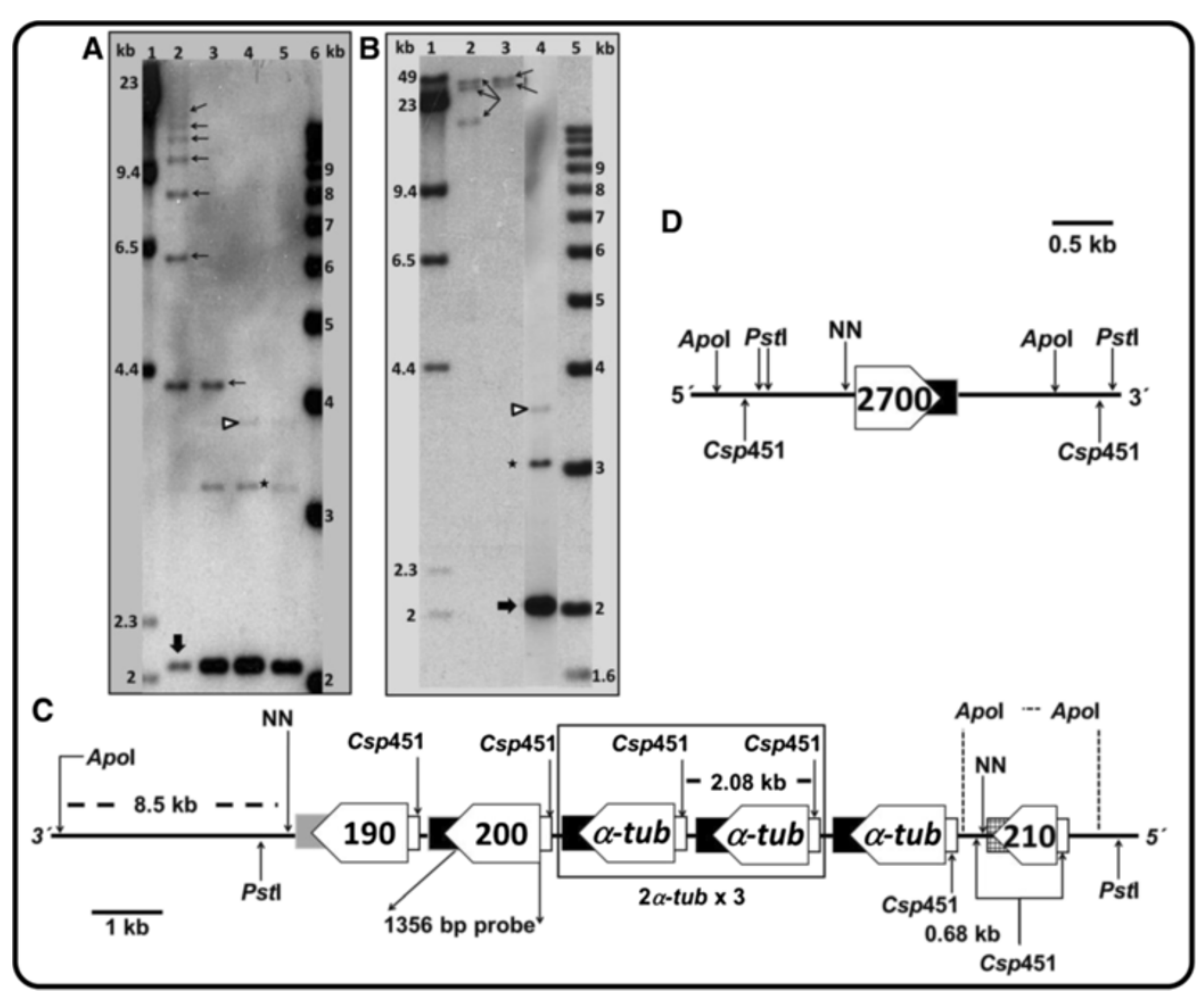

Figure 2 Genomic organization of $L$. braziliensis $\boldsymbol{\alpha}$-tubulin genes. Southern blot of promastigote DNA hybridized with the $\alpha$-tubulin ORF. (A) Two $\mu \mathrm{g}$ of L. braziliensis DNA were partially digested with Csp451 for 2 min (lane 2); 5 min (lane 3) and 15 min (lane 4); lane 5 contains $1 \mu \mathrm{gg}$ of totally digested DNA. Thin arrows in panel A point to the ladder bands; a thick arrow (in panels A and B) points to the repeated unit; the arrow head and the asterisk (in panels A and B) mark additional hybridization bands. (B) Southern blot corresponding to $3 \mu \mathrm{g}$ of $L$. braziliensis DNA digested with either Pstl (lane 2) or Apol (lane 3); 5 min of film exposure. Lane 4 shows a Southern blot of $3 \mu \mathrm{g}$ of Csp451-digested DNA; 20 min of film exposure. Thin arrows point to hybridization bands. Molecular weight markers: lanes 1, a mixture of undigested and Hindlll-digested DNA from $\lambda$ phage; lanes 6, 1-Kb plus marker (Invitrogen California, USA). The position of the DNA markers in the blot was revealed by including digoxigenin-labeled markers in the hybridization mixture. As probe, the L. braziliensis $\alpha$-tubulin ORF was used. (C) Hypothetical map for the $\alpha$ tubulin locus in L. braziliensis chromosome 13, as deduced from Southern blot analyses and genomic sequence at GeneDB database (D). Map for $\alpha$-tubulin locus in L. braziliensis chromosome 29, as deduced from genomic sequence at GeneDB database. Pentagonal shaped boxes represent $\alpha$-tubulin genes, numbers inside each box are verb GeneDB entries (LbrM.13.0190, LbrM.13.0200, LbrM.13.0210 and LbrM.29.2700); filled boxes at the end of each gene represent the $3^{\prime}$ UTR; and rectangles located at the 5' end of each gene, the 5' UTR.

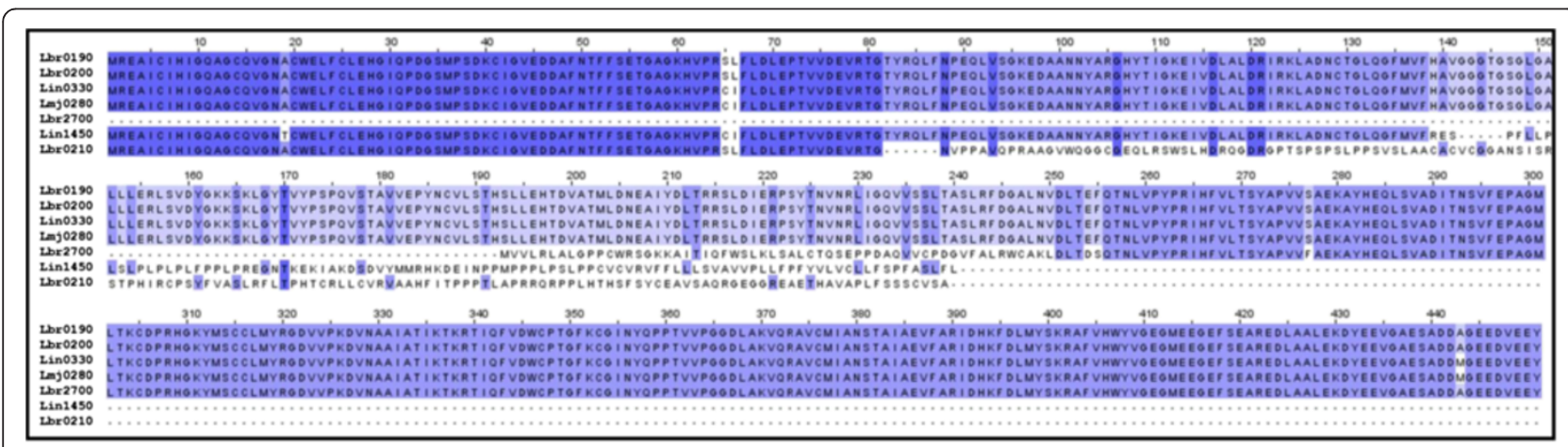

Figure 3 Comparison of $\boldsymbol{\alpha}$-tubulin sequences among Leishmania species. Multiple sequence alignment of LbrM.13.0190 (Lbr0190), LbrM.13.0200 (Lbr0200), LinJ.13.0330 (Lin0330), LmjF.13.0280 (Lbr0280), LbrM.29.2700 (Lbr2700), LinJ.13.1450 (Lin1450) and LbrM.13.0210 (Lbr0210) deduced amino acid sequences, constructed using Clustal-W and Jalview tools. 
MHOM/BR/75/M2903 strain [20], in other Leishmania species, even though several genomes have been sequenced (L. panamensis, $L$. major, $L$. infantum, $L$. mexicana, $L$. donovani and L. tarentolae), or in related trypanosomatids (Trypanosoma brucei and T. cruzi). Finally, an additional finding pointing to a possible assembly error in gene LbrM.13.0210 is the fact that the region conserved with $\alpha$-tubulin would extend further (35 amino acids) if the nucleotide at position 245 is deleted (data not shown).

\section{The $L$. braziliensis $\alpha$-tubulin locus at chromosome 13 contains two types of $\alpha$-tubulin genes differing in their 3' UTR}

Given that Leishmania genes are transcribed into polycistronic RNA precursors that need to be further processed into individual mRNAs by trans-splicing and polyadenylation, post-transcriptional regulation represents the main level of controlling gene expression in these parasites $[8,21]$. Consequently, regulatory sequences located in the $5^{\prime}$ and $3^{\prime}$ untranslated region (UTR) are involved in the modulation of mRNA processing, mRNA stabilization/destabilization, mRNA halflife, or translation efficiency $[8,21]$. To determine the UTRs for $L$. braziliensis $\alpha$-tubulin gene, RT-PCR reactions using specific oligonucleotides were performed. Thus, it was possible to determine the 5' UTR region of the $\alpha$-tubulin genes LbrM.13.0190 and LbrM.13.0200, which was 69 nucleotides in length and identical for both genes. This sequence was deposited in the GenBank database under the accession number FJ750454, and it was incorporated to the GeneDB database [22]. Regarding the 3' UTR, by RT-PCR we determined that this region for LbrM.13.0200 (and probably for other genes present in the locus, see below) is 369-nucleotide in length (GenBank accession number FJ750455); henceforth this sequence will be referred as 3' UTR-I. On the other hand, the 3' UTR of the last gene of the cluster (i.e. LbrM.13.0190), referred here as 3'UTR-II, was found to be highly divergent (Figure 4A). Taking into account that the L. braziliensis $\alpha$-tubulin locus at chromosome 13 should be composed by $8-15$ copies of the prototypic $\alpha$-tubulin gene, but they collapsed to only two (LbrM.13.0190 and LbrM.13.0200) during assembly process, it is reasonable concluding that the 3' UTR for the rest of genes would be identical or near-identical to the 3' UTR-I. Similar situation has been reported in $\beta$ tubulin array from L. major [9], and HSP7O genes [17] in which the last gene of the tandem bears a 3' UTR different to that found in the rest of genes. The distinctive genomic environment provided by each 3' UTR type (I and II) might obey to a need of providing specific expression profiles that ensure the availability of the $\alpha$-tubulin protein under different conditions as occurs with the HSP70 protein [23]. Remarkably, bioinformatics analysis of these two sequences showed that whereas the 3' UTR-I (LbrM.13.0200 gene) has a PRE motif (Figure 4B), which has been implicated in the promastigote-specific expression of several genes [24], the 3' UTR-II (LbrM.13.0190 gene) has an ARE motif (Figure $4 \mathrm{C}$ ), which is recognized as an mRNA instability element [25].

\section{The mRNA levels for L. braziliensis $\alpha$-tubulin are controlled post-transcriptionally by mechanisms sensitive to changes in environmental temperature}

Analysis of $\alpha$-tubulin gene expression in L. braziliensis has not been addressed to date. Early studies in Leishmania enriettii demonstrated that in agreement with the greater tubulin content in the promastigote stage, the levels of $\alpha$ - and $\beta$-tubulin mRNAs in the promastigotes were significantly higher than those found in amastigotes [26]. In L. major, using microarray hybridizations, it was found that $\alpha$-tubulin mRNAs are 6.3-fold more abundant in promastigotes than in amastigotes [27]. However, in L. mexicana, similar amounts of tubulin mRNAs were found in both promastigotes and amastigotes $[28,29]$. In the related trypanosomatid $T$. cruzi, the regulation of tubulin genes has been studied in some extent [30,31]. In this parasite, lower levels of the $\alpha$ - and $\beta$ tubulin transcripts are found in amastigotes and trypomastigotes compared to epimastigotes. Experimental evidence suggests tubulin mRNA half-lives are negatively affected by the amount of free, unpolimerized tubulin proteins. The regulatory mechanism would operate through the recognition of sequence elements located at the beginning of the coding region and in the $3^{\prime}$ UTR of the tubulin genes [31].

Temperature shifts trigger differential gene expression and stage transformation in Leishmania [32]. Thus, we first examined the effect of heat shock treatment on the L. braziliensis $\alpha$-tubulin mRNA stability. For this purpose, parasites were cultured at $35^{\circ} \mathrm{C}$ during several periods of time, determining the parasite morphology and $\alpha$-tubulin mRNA abundance at each time culture-point. As shown in Figure 5, as incubation time at $35^{\circ} \mathrm{C}$ was increased, clear alterations in the parasite morphology were observed; parasites turned into rounded shape forms. Interestingly, a concomitant decrease in the $\alpha$ tubulin mRNA abundance was also observed, suggesting a down-regulation of $\alpha$-tubulin mRNAs levels during adaptation of the parasite to the mammalian host temperature.

In order to obtain clues about the regulatory mechanisms regulating mRNA stability of the L. braziliensis $\alpha$-tubulin genes, we used inhibitors of transcription 


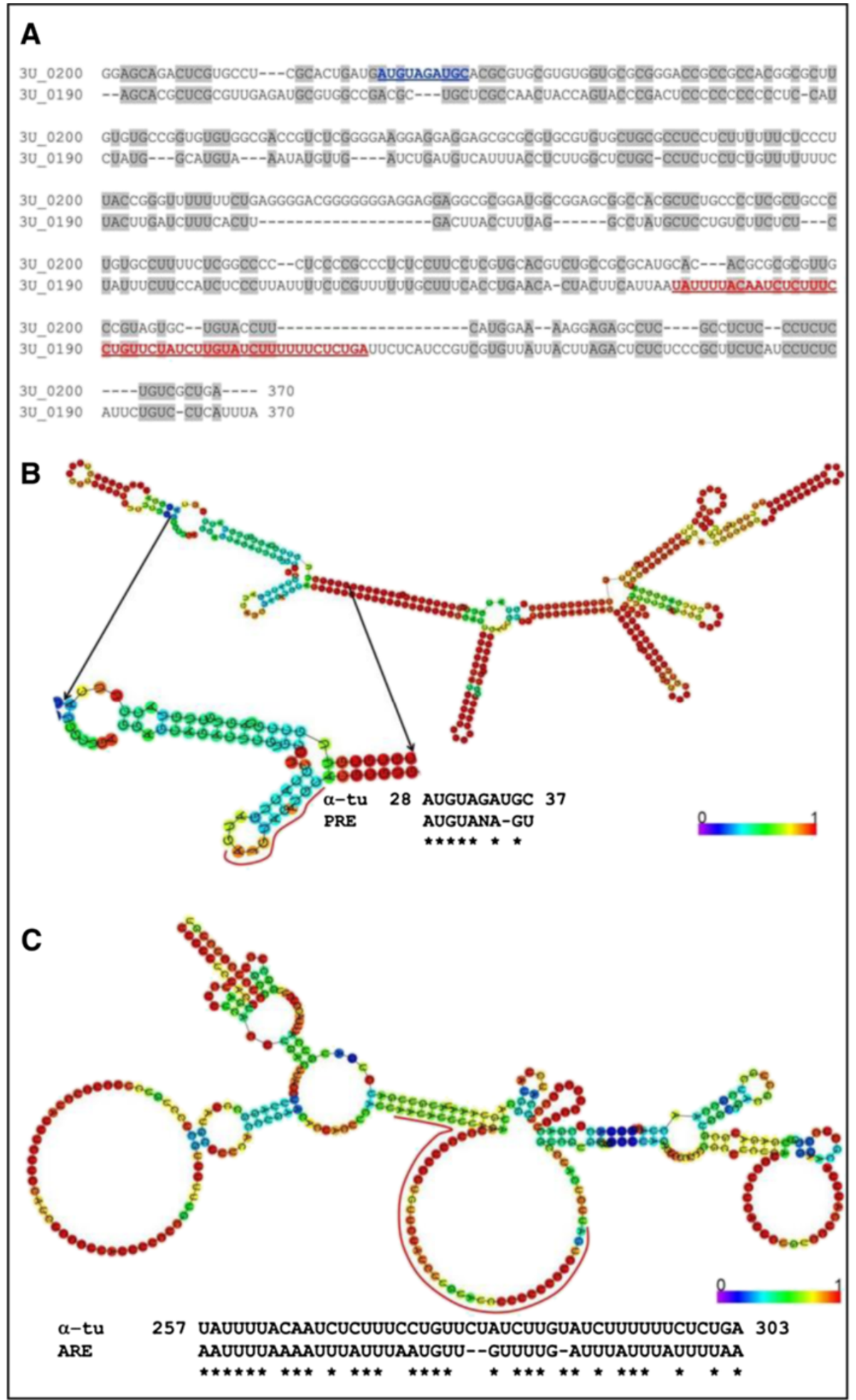

Figure 4 Analysis of the $\mathbf{3}^{\prime}$ UTR of $\boldsymbol{\alpha}$-tubulin genes from Leishmania braziliensis. (A) Alignment between the 3' UTR-I (LbrM.13.0200) and the 3' UTR-II (LbrM.13.0190). (B) PRE element (highlighted in blue in A) identified in the 3' UTR-I. (C) ARE element (highlighted in red in A) observed in the $3^{\prime}$ UTR-II. The color scale in B and C denotes the structure probability from blue (0) to red (1), according to [44]. 


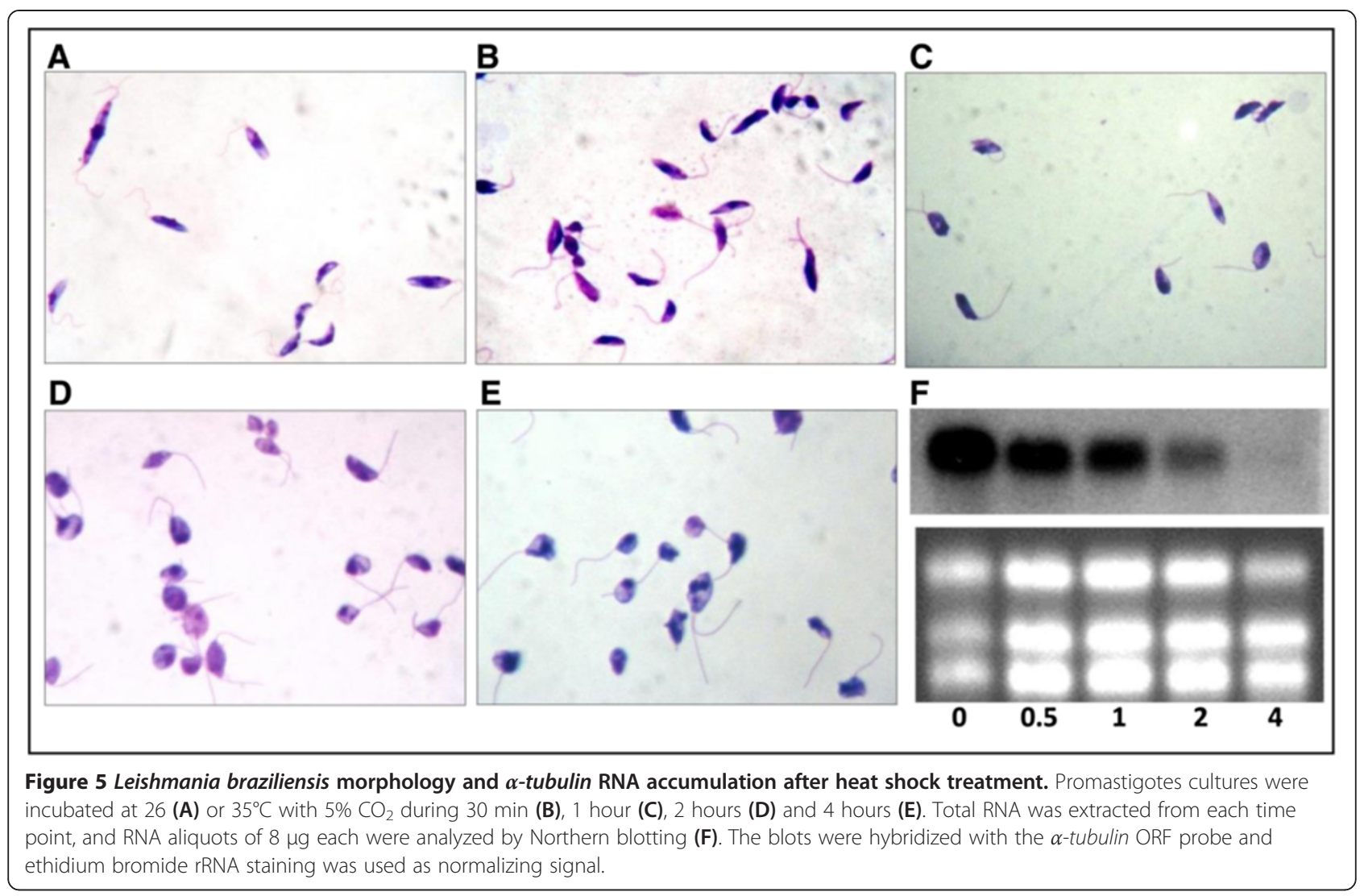

(actinomycin D), trans-splicing (sinefungin) and protein synthesis (cycloheximide). The effects of these drugs were analyzed at both $26^{\circ} \mathrm{C}$ and $35^{\circ} \mathrm{C}$ for several periods of time (Figure 6). As expected, inhibition of transcription and mRNA processing at $26^{\circ} \mathrm{C}$, led to a continuous decrease in the $\alpha$-tubulin mRNAs (Figure 6, panel A). The decrease was more dramatic in parasites incubated at $35^{\circ} \mathrm{C}$ (Figure 6, panel C), suggesting the existence of a destabilizing mechanism operating at this temperature. A support for the existence of such a specific mechanism came from the observation that protein synthesis inhibition of $L$. braziliensis promastigotes at $35^{\circ} \mathrm{C}$ (Figure 6, panel $\mathrm{D}$ ) but not at $26^{\circ} \mathrm{C}$ (Figure 6, panel B), led to an increase of the $\alpha$ tubulin mRNA half-lives. Moreover, protein synthesis inhibition at $26^{\circ} \mathrm{C}$ (Figure 6, panel B) seems to have no effect on the decay rate of the $\alpha$-tubulin mRNAs (Figure 6, panel A). Thus, it can be hypothesized the existence of a labile protein factor, induced during promastigote to amastigote differentiation, that is responsible of decreasing the $\alpha$-tubulin mRNA half-lives in the mammalian stage of L. braziliensis. This finding would be in agreement with the fact, known since the 1980 decade, that tubulins are more abundant proteins in the promastigote stage than in the amastigote one $[5,28,29]$.
Gene expression in trypanosomatids is controlled by post-transcriptional mechanisms relying on the modulation of mRNA processing, mRNA stabilization/destabilization, mRNA half-life, translation efficiency or post-translational modifications $[8,21]$. Accordingly, a variety of elements in the untranslated regions (UTR) have been shown to regulate these events [33-35]. Now, in this study, the genomic organization and the structure of $5^{\prime}$ and 3' UTRs for $\alpha$-tubulin genes have been determined. Next step will be to investigate the involvement of the motifs contained in the 3' UTR-I and -II sequences in the regulatory mechanisms controlling tubulin expression in L. braziliensis.

\section{Conclusions}

The analysis of the genomic organization and genome distribution of $\alpha$-tubulin genes in several Leishmania species suggests that these genes have been reorganized in a drastic manner along Leishmania speciation. Nevertheless, the existence of a gene cluster, consisting of several copies of tandemly arranged $\alpha$-tubulin genes, was found to be a common feature in the different Leishmania species. In the L. braziliensis tandem array, two types of $\alpha$-tubulin genes differing only in their 3' UTR were characterized: most of the $\alpha$-tubulin gene copies have an identical 3' UTR (named 3' UTR-I), whereas 


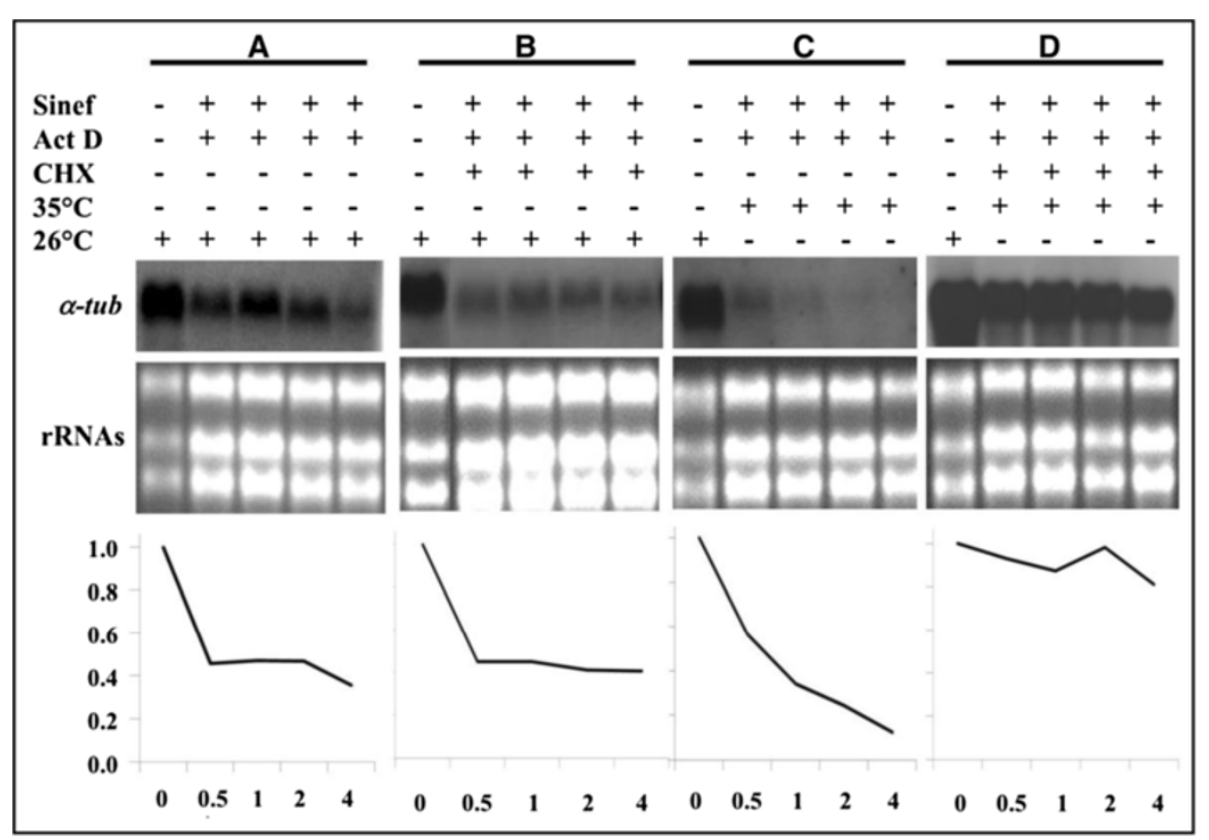

Figure 6 Heat shock treatment and protein synthesis inhibition effect on the stability of $\boldsymbol{\alpha}$-tubulin transcripts. For total transcription inhibition, promastigotes cultured at $26^{\circ} \mathrm{C}$ were incubated with $10 \mu \mathrm{g} / \mathrm{ml}$ sinefungin (Sinef) five minutes previously to the addition of $10 \mu \mathrm{g} / \mathrm{ml}$ actinomycin D (Act D). Afterwards, cultures were incubated either at $26^{\circ} \mathrm{C}(\mathbf{A})$ or $35^{\circ} \mathrm{C}$ with $5 \% \mathrm{CO}_{2}$ (C) for $0,0.5,1,2$, and 4 hours. For protein synthesis inhibition, $20 \mathrm{\mu g} / \mathrm{ml}$ of cycloheximide (CHX) was used in promastigotes cultured either at $26^{\circ} \mathrm{C}$ (B) or $35^{\circ} \mathrm{C}$ with $5 \% \mathrm{CO}_{2}$ (D) for $0,0.5,1$, 2, and 4 hours. Finally, total RNA was extracted, and $8 \mu \mathrm{g}$ from each sample were analyzed by Northern blotting. The blots were hybridized with $\alpha$-tubulin ORF probe. Before transferring, gels were stained with ethidium bromide and photographed; rRNA staining was used as normalizing signal for densitometric measurement. For each series, densitrometric graphs (bottom panels) were standardized against the RNA signal found at time $0\left(26^{\circ} \mathrm{C}\right)$, which was arbitrarily set as 1.0 .

the last copy of the tandem possesses a different sequence (named 3' UTR-II). Finally, we demonstrated that mRNA expression of L. braziliensis $\alpha$-tubulin genes is controlled by a temperature-dependent posttranscriptional mechanism.

\section{Methods}

\section{Parasite cultures and nucleic acids extraction}

Promastigotes of L. braziliensis MHOM/BR/75/M2904 were cultured in vitro either at $26^{\circ} \mathrm{C}$ in Schneider's insect medium (Sigma Aldrich, Inc., St. Louis, USA) supplemented with 20\% heat-inactivated fetal calf serum (Eurobio, Inc., Les Ulis, France), and $0.1 \mu \mathrm{g} / \mathrm{mL}$ of 6-biopterin (Sigma Aldrich,Inc., St. Louis, USA) or at $35^{\circ} \mathrm{C}$ with $5 \% \mathrm{CO}_{2}$ by several time periods. Total DNA and RNA from parasite cells were isolated using the phenolchloroform-isoamilic alcohol method [17], and the TRIzol method (Invitrogen, California, USA) according to manufacturer instructions, respectively.

\section{Oligonucleotides}

All oligonucleotides were synthesized by IDT, Inc. (Miami, USA). The L. braziliensis $\alpha$-tubulin coding region was amplified from genomic DNA by PCR with Lb-Tub-F (5' GGATCCATGCGTGAGGCTATCTGC
3') and Lb-Tub-R (5' CTGCAGCTAGTACTCCTC GACGTCCT $3^{\prime}$ ) primers, which contain the BamHI and Pst I restriction sites (underlined in the sequence), respectively. Amplification of the UTRs was performed from poly-T primed-cDNA using the following primers: LbSL (5' CGCTATATAAGTATCAGTTTC 3') and Lb $\alpha$ T-106 (5' GTGAGGCTATCTGCATTCA 3') for the 5' UTR and Lb $\alpha$ t1312 (5' TCGTGCACTGGTACGTTG $3^{\prime}$ ) and poly-T EcoRI (5' CGGAATTCTTTTTTTTTT TTTTTTTTT 3'), for the 3' UTR.

\section{Cloning sequences and in silico analyses}

First-strand cDNA synthesis was carried out from $L$. braziliensis total RNA using an oligo-dT primer and the Transcriptor first strand cDNA synthesis kit (Roche, Inc., Mannheim, Germany). The following PCR mix reaction was used for all amplifications in a final volume of $20 \mu \mathrm{l}: 1 \times$ reaction buffer $(10 \mathrm{mM}$ Tris- $\mathrm{HCl} \mathrm{pH}$ 9.0, 50 $\mathrm{mM} \mathrm{KCl}, 0.1 \%$ Triton X-100), $1.5 \mathrm{mM} \mathrm{MgCl}_{2}, 0.4 \mathrm{mM}$ of dNTP mix, $0.5 \mu \mathrm{M}$ of each primer, $1 \mathrm{M}$ betain, 0.06 units per $\mu \mathrm{l}$ of expand high fidelity enzyme (Roche, Inc., Mannheim, Germany) and $15 \mathrm{ng} / \mu \mathrm{l}$ DNA or $1 \mu \mathrm{l}$ of cDNA product. An MJ Research PTC-100 DNA thermocycler was used for the reaction with the following amplification profile: $95^{\circ} \mathrm{C} / 5 \mathrm{~min}$ (initial denaturation), 35 cycles at 
$92^{\circ} \mathrm{C} / 0.5 \mathrm{~min}$, annealing at $52-58^{\circ} \mathrm{C} / 0.5 \mathrm{~min}$ and extension for $72^{\circ} \mathrm{C} / 1 \mathrm{~min}$, with a final incubation at $72^{\circ} \mathrm{C}$ for $10 \mathrm{~min}$. All the amplified fragments were resolved in agarose gels and visualized under UV exposure after ethidium bromide staining. RT-PCR products were excised from gels, purified using Wizard ${ }^{\circ}$ SV Gel and PCR Clean-Up System (Promega, Inc., Madison, WI, USA) and cloned into the $\mathrm{pCR}^{\oplus}$ II (Invitrogen, California, USA) or pGEM ${ }^{\oplus} \mathrm{T}$ Easy plasmids (Promega, Inc., Madison, WI, USA). The following clones were obtained and submitted to the Addgene web site [36] pTOLb $\alpha$ tub-B (Addgene ID, 42937), containing the $\alpha$-tubulin ORF, pLb5 $\alpha$ Tub-D (Addgene ID, 42938), the 5' UTR and pLb3 $\alpha$ Tub-C6 (Addgene ID, 42939), the 3' UTR. The sequences from each plasmid insert were determined using the Big Dye Terminators v3.1 kit (Applied Biosystem, California, USA) by automatic sequencing at the Servicio de Genómica (Parque Científico de Madrid, Universidad Autónoma de Madrid). In order to deduce the $\alpha$-tubulin mRNA UTRs from other Leishmania species, LALIGN [37], and ClustalW analyses [38] were carried out. Gene comparison for investigating the constitution of $\alpha$-tubulin variants was performed by BLAST/N analysis [20]. Data from TriTrypDB [39] were retrieved for studying the $\alpha$ tubulin loci synteny and comparison among the three Leishmania species was carried out by BLAST/N analysis.

\section{Southern blot analyses}

With the aim of choosing endonucleases cutting at the boundaries of the $\alpha$-tubulin locus (Pst I and ApoI) or inside it (Csp451), sequences of $\alpha$-tubulin loci from the three Leishmania species were retrieved from GeneDB database [40] and used in an in silico restriction analysis through NEBcutter V2.0 tools [41]. DNA from promastigotes was totally or partially digested with the selected restriction enzymes according to the manufacturer specifications (Promega, Inc., Madison, WI, USA). Resulting fragments were electrophoresed during sixteen to eighteen hours at 50 volts on $25 \mathrm{~cm}$ low electroendosmosis agarose gel at $0.8 \%$ (Ambion, Inc., Texas, USA), transferred to a nylon membrane (Roche, Inc., Mannheim Germany) by the saline-sodium citrate (SSC) buffer method [42] and hybridized with the $\alpha$ tubulin ORF as probe. For probe synthesis, the $L$. braziliensis $\alpha$-tubulin gene was released from clone pTOLb $\alpha$ tub-B by BamHI/Pst I cutting, excised from gel, purified using Wizard ${ }^{\oplus} \mathrm{SV}$ Gel and PCR Clean-Up System (Promega, Inc., Madison, WI, USA), and finally labeled with digoxigenin by randomly primed synthesis using the DIG High Prime DNA Labeling kit (Roche, Inc., Mannheim, Germany). Hybridizations were performed using the Detection Starter kit II (Roche, Inc., Mannheim, Germany) according to manufacturer's instructions.
Afterwards, membranes were washed twice under the following conditions: $2 \times$ sodium citrate solution (SSC) and $0.5 \%(\mathrm{p} / \mathrm{v})$ sodium dodecyl sulfate (SDS) at room temperature for $5 \mathrm{~min}, 0.5 \times \mathrm{SSC}, 0.1 \%$ SDS solution for $15 \mathrm{~min}$ at $65^{\circ} \mathrm{C}$ and $0.1 \times \mathrm{SSC}, 0.1 \%$ SDS solution for 15 min at $65^{\circ} \mathrm{C}$, under constant shaking. Finally, after the immunological detection according to the above mentioned kit recommendations, membranes were exposed on Curix RP2 plus medical X-Ray film (AGFA, Mortsel, Belgium).

\section{Northern blot analyses}

To investigate the $\alpha$-tubulin expression levels, $L$. braziliensis promastigote cultures were treated as follows: maturation of mRNA were inhibited by addition of $10 \mu \mathrm{g} / \mathrm{ml}$ sinefungin to the $L$. braziliensis promastigote cultures at logarithmic phase $\left(5-9 \times 10^{6}\right.$ promastigotes per $\mathrm{ml}$ ), synthesis of RNA were inhibited by addition of $10 \mu \mathrm{g} / \mathrm{ml}$ actinomycin D five min later [43]. Protein synthesis inhibition was achieved by adding $10 \mu \mathrm{g} / \mathrm{ml}$ of cycloheximide on promastigote cultures simultaneously treated with sinefungin and actinomycin D. Parasites were treated at 26 or $35^{\circ} \mathrm{C}$ with $5 \% \mathrm{CO}_{2}$, with or without inhibitors for several time periods (0.5, 1, 2 and 4 hours). Afterwards, parasites were harvested for RNA extraction and $8 \mu \mathrm{g}$ of total RNA per line were separated on $1.5 \%(\mathrm{w} / \mathrm{v})$ low electroendosmosis agarose/MOPS/ formaldehyde gels (Ambion, Inc., Texas, USA) and transferred to nylon membranes (Roche, Inc., Mannheim, Germany). Probes, hybridization and immunological detection were performed as previously mentioned.

\section{Additional file}

Additional file 1: Figure S1. (A) The $3^{\prime}$ end of LbrM.13.0210 gene is derived from chromosome 28. In the intercoding region of LbrM.28.2580 and LbrM.28.2590 entries at chromosome 28 from L. braziliensis there is a 457 nucleotide sequence that is identical to the final 355 nucleotides of the LbrM.13.0210 ORF and the first 102 downstream nucleotides. Boxes in red comprise ORFs. (B) Alignment of the LbrM.13.0210 entry with the intergenic region between the LbrM.28.2580 and the LbrM.28.2590 entries (28IGR). A $100 \%$ of identity in $457 \mathrm{nt}$ overlapped, showed in red, was found. The ATG and stop codons of the LbrM.13.0210 entry are indicated in blue

\section{Competing interests}

The authors declare that they have no competing interests.

\section{Authors' contributions}

CAR, JMR, and CJP conceived and designed the experiments. CAR performed the experiments. CAR, JMR, and CJP analyzed the data. CAR, JMR, and CJP wrote the paper. All authors revised and approved the final version of the manuscript.

\section{Acknowledgments}

CJP's lab was supported by Pontificia Universidad Javeriana (Colombia), Research project ID PPTA 00003780. JMR's lab was supported by grants from the Ministerio de Ciencia y Tecnología (BFU2009-08986) and the Fondo de Investigaciones Sanitarias (ISCIII-RETIC RD12/0018/0009-FEDER). CAR was 
supported by Colciencias, Programa Nacional de Doctorados, convocatoria 2008.

\section{Author details}

'Laboratorio de Parasitología Molecular, Departamento de Microbiología, Facultad de Ciencias, Pontificia Universidad Javeriana, Carrera 7 No. 43-82, Edificio 52, Oficina 608, Bogotá, Colombia. ${ }^{2}$ Centro de Biología Molecular "Severo Ochoa" (CSIC-UAM), Universidad Autónoma de Madrid, 28049, Madrid, Spain.

Received: 8 February 2013 Accepted: 19 June 2013

Published: 6 July 2013

\section{References}

1. McKean PG, Vaughan S, Gull K: The extended tubulin superfamily. J Cell Sci 2001, 114(Pt 15):2723-2733.

2. Gull K: The cytoskeleton of trypanosomatid parasites. Annu Rev Microbiol 1999, 53:629-655.

3. Murray HW, Berman JD, Davies CR, Saravia NG: Advances in leishmaniasis. Lancet 2005, 366(9496):1561-1577.

4. Alvar J, Velez ID, Bern C, Herrero M, Desjeux P, Cano J, Jannin J, den Boer M: Leishmaniasis worldwide and global estimates of its incidence. PLoS One 2012, 7(5):e35671.

5. Fong D, Chang KP: Tubulin biosynthesis in the developmental cycle of a parasitic protozoan, Leishmania mexicana: changes during differentiation of motile and nonmotile stages. Proc Natl Acad Sci U S A 1981, 78(12):7624-7628.

6. Coulson RM, Connor V, Chen JC, Ajioka JW: Differential expression of Leishmania major beta-tubulin genes during the acquisition of promastigote infectivity. Mol Biochem Parasitol 1996, 82(2):227-236.

7. Jackson AP, Vaughan S, Gull K: Evolution of tubulin gene arrays in trypanosomatid parasites: genomic restructuring in Leishmania. BMC Genomics 2006, 7:261.

8. Requena JM: Lights and shadows on gene organization and regulation of gene expression in Leishmania. Front Biosci 2011, 16:2069-2085.

9. Jackson AP, Vaughan S, Gull K: Comparative genomics and concerted evolution of beta-tubulin paralogs in Leishmania spp. BMC Genomics 2006, 7:137.

10. Landfear SM, McMahon-Pratt D, Wirth DF: Tandem arrangement of tubulin genes in the protozoan parasite Leishmania enriettii. Mol Cell Biol 1983, 3(6):1070-1076.

11. Rogers MB, Hilley JD, Dickens NJ, Wilkes J, Bates PA, Depledge DP, Harris D, Her Y, Herzyk P, Imamura H, Otto TD, Sanders M, Seeger K, Dujardin JC, Berriman M, Smith DF, Hertz-Fowler C, Mottram JC: Chromosome and gene copy number variation allow major structural change between species and strains of Leishmania. Genome Res 2011, 21(12):2129-2142.

12. Peacock CS, Seeger K, Harris D, Murphy L, Ruiz JC, Quail MA, Peters N, Adlem E, Tivey A, Aslett M, Kerhornou A, Ivens A, Fraser A, Rajandream MA, Carver T, Norbertczak H, Chillingworth T, Hance Z, Jagels K, Moule S, Ormond D, Rutter S, Squares R, Whitehead S, Rabbinowitsch E, Arrowsmith C, White B, Thurston S, Bringaud F, Baldauf SL, et al: Comparative genomic analysis of three Leishmania species that cause diverse human disease. Nat Genet 2007, 39(7):839-847.

13. Ivens AC, Peacock CS, Worthey EA, Murphy L, Aggarwal G, Berriman M, Sisk E, Rajandream MA, Adlem E, Aert R, Anupama A, Apostolou Z, Attipoe P, Bason N, Bauser C, Beck A, Beverley SM, Bianchettin G, Borzym K, Bothe G, Bruschi CV, Collins M, Cadag E, Ciarloni L, Clayton C, Coulson RM, Cronin A, Cruz AK, Davies RM, De Gaudenzi J, et al: The genome of the kinetoplastid parasite, Leishmania major. Science 2005, 309(5733):436-442.

14. Logan-Klumpler FJ, De Silva N, Boehme U, Rogers MB, Velarde G, McQuillan JA, Carver T, Aslett M, Olsen C, Subramanian S, Phan I, Farris C, Mitra S, Ramasamy G, Wang H, Tivey A, Jackson A, Houston R, Parkhill J, Holden M, Harb OS, Brunk BP, Myler PJ, Roos D, Carrington M, Smith DF, Hertz-Fowler C, Berriman M: GeneDB-an annotation database for pathogens. Nucleic Acids Res 2012, 40(Database issue):D98-D108.

15. Oddone R, Schweynoch C, Schonian G, de Sousa CS, Cupolillo E, Espinosa D, Arevalo J, Noyes H, Mauricio I, Kuhls K: Development of a multilocus microsatellite typing approach for discriminating strains of Leishmania (Viannia) species. J Clin Microbiol 2009, 47(9):2818-2825.
16. Spithill TW, Samaras N: Genomic organization, chromosomal location and transcription of dispersed and repeated tubulin genes in Leishmania major. Mol Biochem Parasitol 1987, 24(1):23-37.

17. Ramirez CA, Requena JM, Puerta CJ: Identification of the HSP70-Il gene in Leishmania braziliensis HSP70 locus: genomic organization and UTRs characterization. Parasit Vectors 2011, 4:166.

18. Diez H, Thomas MC, Uruena CP, Santander SP, Cuervo CL, Lopez MC, Puerta $\mathrm{CJ}$ : Molecular characterization of the kinetoplastid membrane protein-11 genes from the parasite Trypanosoma rangeli. Parasitology 2005, 130(Pt 6):643-651.

19. Cuervo C, Lopez MC, Puerta C: The Trypanosoma rangeli histone $\mathrm{H} 2 \mathrm{~A}$ gene sequence serves as a differential marker for KP1 strains. Infect Genet Evol 2006, 6(5):401-409.

20. BLAST/N analysis from TritrypDB. http://tritrypdb.org/tritrypdb/showQuestion. do;jsessionid=78874F49E41EB45CEE66649D01FD46E5?questionFullName= UniversalQuestions.UnifiedBlast.

21. Clayton C, Shapira M: Post-transcriptional regulation of gene expression in trypanosomes and leishmanias. Mol Biochem Parasitol 2007, 156(2):93-101.

22. Incorporation of the 5' UTR region from the a-tubulin genes LbrM.13.0190 and LbrM.13.0200 to the GeneDB database. http://www.genedb.org/gene/ LbrM.13.0200?actionName=\%2FQuery\%2FquickSearch\&resultsSize=1\&taxon NodeName=Root.

23. Folgueira C, Quijada L, Soto M, Abanades DR, Alonso C, Requena JM: The translational efficiencies of the two Leishmania infantum HSP70 mRNAs, differing in their 3'-untranslated regions, are affected by shifts in the temperature of growth through different mechanisms. J Biol Chem 2005, 280(42):35172-35183.

24. Holzer TR, Mishra KK, LeBowitz JH, Forney JD: Coordinate regulation of a family of promastigote-enriched mRNAs by the 3'UTR PRE element in Leishmania mexicana. Mol Biochem Parasitol 2008, 157(1):54-64.

25. Di Noia JM, D'Orso I, Sanchez DO, Frasch AC: AU-rich elements in the 3'untranslated region of a new mucin-type gene family of Trypanosoma cruzi confers mRNA instability and modulates translation efficiency. J Biol Chem 2000, 275(14):10218-10227.

26. Landfear SM, Wirth DF: Control of tubulin gene expression in the parasitic protozoan Leishmania enriettii. Nature 1984, 309(5970):716-717.

27. Leifso K, Cohen-Freue G, Dogra N, Murray A, McMaster WR: Genomic and proteomic expression analysis of Leishmania promastigote and amastigote life stages: the Leishmania genome is constitutively expressed. Mol Biochem Parasitol 2007, 152(1):35-46.

28. Fong D, Wallach M, Keithly J, Melera PW, Chang KP: Differential expression of mRNAs for alpha- and beta-tubulin during differentiation of the parasitic protozoan Leishmania mexicana. Proc Natl Acad Sci U S A 1984, 81(18):5782-5786.

29. Fong D, Chang KP: Changes in tubulin mRNAs during differentiation of a parasitic protozoan Leishmania mexicana. Ann N Y Acad Sci 1986, 466:129-131.

30. Urményi TP, De Castro FT, Carvalho JF, De Souza W, Rondinelli E: Transcriptional and post-transcriptional control of tubulin gene expression in Trypanosoma cruzi. DNA Cell Biol 1992, 11(2):101-109.

31. da Silva RA, Bartholomeu DC, Teixeira SM: Control mechanisms of tubulin gene expression in Trypanosoma cruzi. Int J Parasitol 2006, 36(1):87-96.

32. Alcolea PJ, Alonso A, Gómez MJ, Moreno I, Domínguez M, Parro V, Larraga $V$ : Transcriptomics throughout the life cycle of Leishmania infantum: high down-regulation rate in the amastigote stage. Int J Parasitol 2010, 40 (13):1497-1516.

33. Matthews KR, Tschudi C, Ullu E: A common pyrimidine-rich motif governs trans-splicing and polyadenylation of tubulin polycistronic pre-mRNA in trypanosomes. Genes Dev 1994, 8(4):491-501.

34. Bartholomeu DC, Silva RA, Galvao LM, El-Sayed NM, Donelson JE, Teixeira SM: Trypanosoma cruzi: RNA structure and post-transcriptional control of tubulin gene expression. Exp Parasitol 2002, 102(3-4):123-133.

35. Gupta SK, Carmi S, Waldman Ben-Asher H, Tkacz ID, Naboishchikov I, Michaeli S: Basal splicing factors regulate the stability of mature mRNAs in trypanosomes. J Biol Chem 2013, 288(7):4991-5006.

36. Addgene web site. http://www.addgene.org/.

37. LALIGN a tool for aligning sequences. http://www.ch.embnet.org/software/ LALIGN_form.html.

38. ClustalW tool from EBI. http://www.ebi.ac.uk/Tools/msa/clustalw2/.

39. TritrypDB database. http://tritrypdb.org/tritrypdb/.

40. GeneDB database. http://www.genedb.org/Homepage. 
41. NEBcutter V2.0 tool. http://tools.neb.com/NEBcutter2/.

42. Sambrook J, Russel D: Southern hybridization. In Molecular Cloning: A Laboratory Manual.Volumen 1. 3rd edition. New York: Cold Spring Harbor Laboratory Press; 2001:6.33-6.46.

43. Archer SK, Luu VD, de Queiroz RA, Brems S, Clayton C: Trypanosoma brucei PUF9 regulates mRNAs for proteins involved in replicative processes over the cell cycle. PLoS Pathog 2009, 5(8):e1000565.

44. RNAfold tool. http://rna.tbi.univie.ac.at/cgi-bin/RNAfold.cgi.

doi:10.1186/1471-2164-14-454

Cite this article as: Ramírez et al: Alpha tubulin genes from Leishmania braziliensis: genomic organization, gene structure and insights on their expression. BMC Genomics 2013 14:454.

\section{Submit your next manuscript to BioMed Central and take full advantage of:}

- Convenient online submission

- Thorough peer review

- No space constraints or color figure charges

- Immediate publication on acceptance

- Inclusion in PubMed, CAS, Scopus and Google Scholar

- Research which is freely available for redistribution 